\title{
A Sincere Thank You to the Reviewers of Diabetes Spectrum
}

Each year, Diabetes Spectrum calls on many scientific and medical experts in the field of diabetes to critically review submitted manuscripts. The journal's editors and staff sincerely appreciate these reviewers, whose valuable feedback helps to ensure the high quality of the research and review articles published in each issue. We would like to take this opportunity to publicly thank all of the reviewers who have volunteered their time and expertise in the past year (1 November 2016 to 31 October 2017) for their contributions to Diabetes Spectrum.

Jo Ann Ahern
Oluwaranti Akiyode
Anastasia Albanese-O'Neill
Meghan Ariagno
Martha Barnard
Joshua Barzilay
Nicola Basso
Christine Beebe
James Best
Alyson Blum
Tammi Boiko
Patrick Brady
Catherine Brown
Sandra Burke
Amy Campbell
Kam Capoccia
James Chamberlain
Naomi Chaytor
Robert Chilton
Maureen Chomko
Richard Comi
Sarah Corathers
Susan Cornell
Gillian Culbertson
Brianna Czaikowski
Beth Czerwony
Paresh Dandona
Patricia Davidson
Courtney Davis
Kathleen Dermady
Carsten Dirksen
Amy Donihi
Kimberly Driscoll
Nicole Ehrhardt
Roy Eldor
Hope Feldman
Afomia Feleke
Andreas Festa
Gabor Firneisz
Andrea Fischl

James Fitzgerald
Cynthia Fritschi
Martha Funnell
Ana Gallardo-Hernandez
Theresa Garnero
Erum Ghafoor
Elizabeth Hamilton
Deborah Holtorf
Robert Hood
Paul Iaizzo
Meghan Jardine
Jeremy Johnson
Jan Kavookjian
Janet Kelly
Phillip Kim
Joyce Lee
Gayle Lorenzi
Damien Luviano
Shideh Majidi
Melinda Maryniuk
Kimberly McKeirnan
Lauren McKnight
Lisa Meade
Graydon Meneilly
Maureen Monaghan
Christopher Nolan
Katherine O’Neal
Nora Osemene
Scott Pappada
Mary Lou Perry
Kyle Peters
Tunde Peto
Ninfa Purcell
Neesha Ramchandani
David Robbins
Helena Rodbard
Tami Ross
Barry Rovner
Laurajo Ryan
Marissa Salvo

\author{
Andrew Santeusanio \\ Jochen Schneider \\ Jennifer Smith \\ Guy Soo Hoo \\ Condit Steil \\ Garry Steil \\ Arthur Swislocki \\ Karen Tabb \\ Mitali Talsania \\ Robert Tanenberg \\ Ilker Tasci \\ Alyce Thomas \\ Curtis Triplitt \\ Ganesan Venkatasubramanian \\ Chengda Zhang \\ Lin Zhu \\ Anne Ziesmer
}

